\title{
UAV Flight Stability Control Method Based on Steady - state Error Correction0
}

\author{
Lu Qing-Xiu ${ }^{1}$, Lu Xing-Hua ${ }^{1, *}$, Wang Ling-Feng ${ }^{1}$ \\ ${ }^{1}$ Huali College Guangdong University of Technology, 511325 Guangzhou Zengcheng, China
}

\begin{abstract}
UAV flight stability control system is a multivariable, non - linear, strongly coupled system, the steady-state error is easy to occur with the traditional fuzzy control method. An UAV flight stability control method is proposed based on steady - state error correction, the steady state dynamic model of UAV flight is constructed, and the control constraint model is constructed with the roll angle, the pitch angle, the longitudinal inclination angle and the acceleration as the constraint parameters. The construction of UAV flight stability control objective function is obtained, and the linearization treatment is carried out near the equilibrium point of the flight of the unmanned aerial vehicle, and the equivalent control law of the sliding mode surface is constructed. Based on the conventional synovial surface, an integral term of tracking error is added to reduce the steady-state error and realize adaptive correction of flight parameters. The simulation results show that the stability of the flight control of the UAV is better by the method, and the self - adaptive correction capability of the flight state parameters is strong.
\end{abstract}

\section{INTRODUCTION}

As a small aircraft, UAV has good application value in carrying out close distance transportation, target detection and aerial reconnaissance. UAV can be defined as unmanned helicopter from the technical point of view. Unmanned fixed-wing aircraft, unmanned multi-rotor aircraft, unmanned airship, unmanned paratroopterfly, etc. According to the application field, UAV can be divided into military UAV and civilian UAV. The UAV has the characteristics of good maneuverability, strong concealment and low risk coefficient. It will be widely used in military and civil fields in the future[1].

In the course of flight, the unmanned aerial vehicle is affected by the disturbance airflow and the factors of the aircraft itself, and under the interference of uncertain climatic conditions, the flight attitude is unstable, the steady - state error is generated, and the flight stability control is required. The mathematical model of UAV is a dynamic system described by a set of nonlinear differential equations due to the continuous changes of the flight conditions such as atmospheric density. For this kind of system, the traditional flight control methods generally use gain scheduling algorithm, combined with fuzzy control method for UAV flight stability control. The nonlinear mathematical model is linearized in multiple flight states in the flight envelope.[2] With the increase of the control constraint variables, the control stability is not good. The system cannot meet the closed-loop performance requirements. If the nonlinear controller is adopted to control the flight stability of the unmanned aerial vehicle, the interpolation or fitting strategy can be adopted, the least square fitting control is carried out, the flight stability control is carried out in combination with the sliding mode integral control idea, and the controller parameters are continuously changed between the finite linear models[3]. By analyzing the nonlinear dynamics model of ARMA, the global nonlinear controller is obtained by synthesizing each single linear controller, and the flight control of the whole UAV is completed. However, the control method has some problems such as weak anti-interference ability and too much randomness, the control effectiveness of flight stability is not good[4]. With the appearance of adaptive control algorithm, adaptive control is able to solve the control problem of nonlinear dynamic system with uncertain parameters. In order to reduce the steady-state error of UAV flight, an UAV flight stability control method is proposed based on steady - state error correction, the steady state dynamic model of UAV flight is constructed, and the control constraint model is constructed with the roll angle, the pitch angle, the longitudinal inclination angle and the acceleration as the constraint parameters. The construction of UAV flight stability control objective function is obtained, and the linearization treatment is carried out near the equilibrium point of the flight of the unmanned aerial vehicle, and the equivalent control law of the sliding mode surface is constructed. Based on the conventional synovial surface, an integral term of tracking error is added to reduce the steady-state error and realize adaptive correction of flight parameters. Finally, simulation results show that this method has superiority in improving the stability of UAV flight control ability. 


\section{STEADY- STATE DYNAMIC MODEL OF UAV FLIGHT AND ANALYSIS OF CONTROL CONSTRAINTS}

\subsection{Steady - state dynamic model of UAV flight}

The motion equations of the UAV mainly include the motion equation of the centroid and the kinetic equation of the centroid. These equations need to be obtained according to the moment of momentum and the theorem of moment of momentum. In addition, the angular relationship equation is used when coordinate conversion is carried out. These equations can be combined to obtain a closed unmanned aerial vehicle' s equations of space motion. The construction of UAV's flight dynamics model is mainly used in the following coordinate systems[5]: velocity coordinate system $O x_{3} y_{3} z_{3}$, body coordinate system $O x_{1} y_{1} z_{1}$, Ballistic coordinate system $O x_{2} y_{2} z_{2}$, earth fixed axis system $A x y z$, combined with the constraint conditions, three state equations for the flight stability control of UAV can be obtained.:

$$
\begin{aligned}
& \left(M_{R L} R^{2}+J_{R L}+\frac{1}{4} M_{P} R^{2}+\frac{R^{2}}{D^{2}} J_{P \delta}\right) \ddot{\theta}_{R L} \\
& +\left(\frac{1}{4} M_{P} R^{2}-\frac{R^{2}}{D^{2}} J_{P \delta}\right) \ddot{\theta}_{R R}+\frac{1}{2} M_{P} R L \ddot{\theta_{P}}=C_{L} \\
& \left(M_{R R} R^{2}+J_{R R}+\frac{1}{4} M_{P} R^{2}+\frac{R^{2}}{D^{2}} J_{P \delta}\right) \ddot{\theta_{R R}} \\
& +\left(\frac{1}{4} M_{P} R^{2}-\frac{R^{2}}{D^{2}} J_{P \delta}\right) \ddot{\theta_{R L}}+\frac{1}{2} M_{P} R L \ddot{\theta_{P}}=C_{R} \\
& \frac{1}{2} M_{P} R L \ddot{\theta_{R L}}+\frac{1}{2} M_{P} R L \ddot{\theta}_{R R}+\left(J_{P \theta}+M_{P} L^{2}\right) \ddot{\theta_{P}} \\
& =M_{P} g L \theta_{P}-\left(C_{L}+C_{R}\right)
\end{aligned}
$$

We use $X_{R M}, V_{R M}, \theta_{P}, \omega_{P}, \delta, \dot{\delta}$ to represent the state parameters of UAV flight stability control system. The steady state equation of the acceleration of the center of mass of UAV in state space can be calculated as follows:

$$
\left(\begin{array}{c}
X_{R M} \\
V_{R M} \\
\dot{\theta_{P}} \\
\dot{\omega_{P}} \\
\dot{\delta} \\
\ddot{\delta}
\end{array}\right)=\left(\begin{array}{cccccc}
0 & 1 & 0 & 0 & 0 & 0 \\
0 & 0 & A_{23} & 0 & 0 & 0 \\
0 & 0 & 0 & 1 & 0 & 0 \\
0 & 0 & A_{43} & 0 & 0 & 0 \\
0 & 0 & 0 & 0 & 0 & 1 \\
0 & 0 & 0 & 0 & 0 & 0
\end{array}\right)\left(\begin{array}{c}
X_{R M} \\
V_{R M} \\
\theta_{P} \\
\omega_{P} \\
\delta \\
\dot{\delta}
\end{array}\right)+\left(\begin{array}{cc}
0 & 0 \\
B_{2} & B_{2} \\
0 & 0 \\
B_{4} & B_{4} \\
0 & 0 \\
B_{6} & -B_{6}
\end{array}\right)\left(\begin{array}{l}
C_{L} \\
C_{R}
\end{array}\right)
$$

Wherein:

$$
\begin{gathered}
A_{23}=\frac{-M_{P}{ }^{2} L^{2} g}{M_{P} J_{P \theta}+2\left(J_{P \theta}+M_{P} L^{2}\right)\left(M_{R}+J_{R} / R^{2}\right)} \\
A_{43}=\frac{M_{P}{ }^{2} g L+2 M_{P} g L\left(M_{R}+J_{R} / R^{2}\right)}{M_{P} J_{P \theta}+2\left(J_{P \theta}+M_{P} L^{2}\right)\left(M_{R}+J_{R} / R^{2}\right)}
\end{gathered}
$$

$$
\begin{gathered}
B_{2}=\frac{\left(J_{P \theta}+M_{P} L^{2}\right) / R+M_{P} L}{M_{P} J_{P \theta}+2\left(J_{P \theta}+M_{P} L^{2}\right)\left(M_{R}+J_{R} / R^{2}\right)} \\
B_{4}=\frac{-\frac{R+L}{R} M_{P}-2\left(M_{R}+J_{R} / R^{2}\right)}{M_{P} J_{P \theta}+2\left(J_{P \theta}+M_{P} L^{2}\right)\left(M_{R}+J_{R} / R^{2}\right)} \\
B_{6}=\frac{D / 2 R}{J_{P \theta}+\frac{D^{2}}{2 R}\left(M_{R} R+J_{R} / R\right)}
\end{gathered}
$$

The relationship between the resistance, lift and lateral force of the unmanned aerial vehicle in the body coordinate system is: $C=K_{m}\left(U-K_{e} \dot{\theta_{P}}\right)$, wherein $K_{m}$, $K_{e}$ are acceleration of mass and back EMF coefficient of UAV, the formula is taken into the equation of state equations as follows:

$\left[\begin{array}{c}\dot{\mathbf{x}} \\ \dot{\mathbf{v}} \\ \dot{\boldsymbol{\theta}} \\ \dot{\boldsymbol{\omega}} \\ \dot{\boldsymbol{\delta}} \\ \ddot{\boldsymbol{\delta}}\end{array}\right]=\left[\begin{array}{ccccll}0 & 1 & 0 & 0 & 0 & 0 \\ 0 & -2 K_{m} K_{e} b_{2} / R & a_{23} & 2 K_{m} K_{e} b_{2} & 0 & 0 \\ 0 & 0 & 0 & 1 & 0 & 0 \\ 0 & -2 K_{m} K_{e} b_{4} / R & a_{43} & 2 K_{m} K_{e} b_{4} & 0 & 0 \\ 0 & 0 & 0 & 0 & 0 & 1 \\ 0 & 0 & 0 & 0 & 0 & -D K_{m} K_{e} b_{6} / R\end{array}\right]\left[\begin{array}{c}\mathbf{x} \\ \mathbf{v} \\ \boldsymbol{\theta} \\ \boldsymbol{\omega} \\ \boldsymbol{\delta} \\ \dot{\boldsymbol{\delta}}\end{array}\right]+\left[\begin{array}{cc}0 & 0 \\ K_{m} b_{2} & K_{m} b_{2} \\ 0 & 0 \\ K_{m} b_{4} & K_{m} b_{4} \\ 0 & 0 \\ K_{m} b_{6} & -K_{m} b_{6}\end{array}\right]\left[\begin{array}{l}\mathbf{U}_{\mathbf{L}} \\ \mathbf{U}_{\mathbf{R}}\end{array}\right]$

It can be seen that the flight status $\delta, \dot{\delta}$ to $X_{R M}, V_{R M}, \theta_{P}, \omega_{P}$ of the UAV are not related to each other, the control system can be decoupled to obtain two separate control subsystems:

$$
\begin{gathered}
{\left[\begin{array}{c}
\mathbf{x}_{\mathbf{m}} \\
\dot{\mathbf{v}_{\mathbf{m}}} \\
\dot{\boldsymbol{\theta}} \\
\dot{\boldsymbol{\omega}}
\end{array}\right]=\left[\begin{array}{cccc}
0 & 1 & 0 & 0 \\
0 & -2 K_{m} K_{e} b_{2} / R & a_{23} & 2 K_{m} K_{e} b_{2} \\
0 & 0 & 0 & 1 \\
0 & -2 K_{m} K_{e} b_{4} / R & a_{43} & 2 K_{m} K_{e} b_{4}
\end{array}\right]\left[\begin{array}{c}
x_{m} \\
v_{m} \\
\theta \\
\omega
\end{array}\right]+\left[\begin{array}{c}
0 \\
K_{m} b_{2} \\
0 \\
K_{m} b_{4}
\end{array}\right] \mathbf{U}_{\boldsymbol{\theta}}} \\
{\left[\begin{array}{c}
\dot{\boldsymbol{\delta}} \\
\ddot{\boldsymbol{\delta}}
\end{array}\right]=\left[\begin{array}{ccc}
0 & 1 \\
0 & -D K_{m} K_{e} b_{6} / R
\end{array}\right]\left[\begin{array}{l}
\delta \\
\dot{\boldsymbol{\delta}}
\end{array}\right]+\left[\begin{array}{c}
0 \\
K_{m} b_{6}
\end{array}\right] \mathbf{U}_{\boldsymbol{\delta}}}
\end{gathered}
$$

Assuming that the longitudinal motion of UAV is symmetrical, a UAV flight dynamics system described by a nonlinear differential equation is constructed[6], and the steady state dynamics model of UAV flight is built.

\subsection{Control constraint parameter analysis}

In order to reduce the steady-state error, the roll angle, pitch angle, longitudinal inclination angle and acceleration are taken as constraint parameters for some extreme conditions, such as the severe change of attitude angle[7]. The control constraint model of UAV flight stability control is constructed, and the piecewise linearization fitting form of flight stability control constraint parameter is obtained:

$$
\left\{\begin{array}{l}
\dot{x}_{1}=x_{3} \\
\dot{x}_{3}=f_{\theta}(X, t)+g_{\theta}(X, t) u(t)+d_{\theta}(t) \\
\dot{x}_{2}=x_{4} \\
\dot{x}_{4}=f_{x}(X, t)+g_{x}(X, t) u(t)+d_{x}(t)
\end{array}\right.
$$


Wherein, $\quad X=[\theta, x, \dot{\theta}, \dot{x}]^{T}, \quad f_{x}(X, t), \quad f_{\theta}(X, t), \quad g_{x}(X, t)$, $g_{\theta}(X, t)$ are gain scheduling control parameters, $d_{x}(t)$, $d_{\theta}(t)$ are fuzzy constraint Inertial parameters considering the existence of external interference. Under the condition of limited initial state, the uncertainty of UAV flight stability control is divided into parameter uncertainty and unmodeled dynamic characteristic uncertainty[8-10]. The unsteady constraint parameters are obtained as follows:

$$
\left\{\begin{array}{c}
s_{x}=\left(\dot{e}_{x}+\lambda_{x} e_{x}+\alpha_{x} \sigma_{x}-e_{x}(0)-\lambda_{x} e_{x}(0)\right) \\
s_{\theta}=\left(\dot{e}_{\theta}+\lambda_{\theta} e_{\theta}+\beta_{\theta} \sigma_{\theta}-\dot{e}_{\theta}(0)-\lambda_{\theta} e_{\theta}(0)\right) \\
S=\lambda s_{x}+s_{\theta}
\end{array}\right.
$$

Same, define $\sigma_{i}$ shows $\sigma_{x}$ and $\sigma_{\theta}, e_{i}$ shows $e_{x}$ and $e_{\theta}$, and:

$$
\begin{gathered}
\dot{\sigma}_{i}=\left\{\begin{array}{c}
\mu \sin \frac{\pi e}{2 \mu},\left|e_{i}\right|<\mu \\
\mu,\left|e_{i}\right| \geq \mu \\
-\mu,\left|e_{i}\right| \leq-\mu
\end{array}\right. \\
u_{e q x}=\lambda\left(-\hat{f}_{x}-\lambda_{x} \dot{e}_{x}-\alpha_{x} \dot{\sigma}_{x}+\ddot{x}_{d}\right) /\left(\lambda g_{x}+g_{\theta}\right) \\
u_{e q_{\theta}}=\left(-\hat{f}_{\theta}-\lambda_{\theta} \dot{e}_{\theta}-\beta_{\theta} \dot{\sigma}_{\theta}+\ddot{\theta}_{d}\right) /\left(\lambda g_{x}+g_{\theta}\right) \\
u_{s w}=-K s a t(S(t) / \mu) \\
u=u_{e q x}+u_{e q \theta}+u_{s w}
\end{gathered}
$$

Through the above treatment, certain parameters of the system are continuously measured in the actual flying process of the unmanned aerial vehicle, and a constraint parameter model of the flight stability control of the unmanned aerial vehicle is constructed.

\section{OPTIMIZATION OF FLIGHT STABILITY CONTROL ALGORITHM FOR UAV}

\subsection{Equivalent control law construction}

Based on the steady - state dynamics model of UAV flight, a flight stability control method based on steady state error correction is presented in this paper. The objective function of UAV flight stability control is constructed. The linearization process is carried out in the vicinity of the equilibrium point of the UAV flight, and the pitching angle tracking error is defined:

$$
e_{1}=\vartheta-\vartheta_{r}
$$

Calculate the derivative of the pitching angle error of UAV flight:

$$
\dot{e}_{1}=\omega_{2}-\dot{\vartheta}_{r}
$$

In order to ensure the proper stability of the pitching angle tracking error, the design of the Lyapunov function and the virtual control amount takes the angular velocity as the virtual control input. At this time, the stability control function is selected as the virtual control input:

$$
\omega_{2 r}=-c_{1} e_{1}+\dot{\vartheta}_{r}-\lambda_{1} \zeta_{1}
$$

Wherein, $c_{1}, \lambda_{1}$ are all greater than zero. When the $\mathrm{UAV}$ is in accelerated motion, the original data will control the executive mechanism of UAV acquisition of the linear weighting and feature extraction, the introduction of integral item definition $\zeta_{1}=\int_{0}^{t} e_{1}(\tau) d(\tau)$. The tracking error of the centroid acceleration of unmanned aerial vehicle is obtained:

$$
\begin{aligned}
& e_{2}=\omega_{2}-\omega_{2 r}=\omega_{2}+c_{1} e_{1}+\lambda_{1} \zeta_{1}-\dot{\vartheta}_{r} \\
& \dot{e}_{1}=e_{2}-c_{1} e_{1}-\lambda_{1} \zeta_{1}
\end{aligned}
$$

And:

The longitudinal motion of the UAV is selected as the Lyapunov function is:

$$
V_{1}=\frac{1}{2} e_{1}^{2}
$$

The longitudinal movement of the UAV Lyapunov function derivative:

$$
\dot{V}_{1}=e_{1} e_{2}-c_{1} e_{1}^{2}-e_{1} \lambda_{1} \zeta_{1}
$$

And:

$$
\dot{e}_{2}=\dot{\omega}_{2}-\dot{\omega}_{2 r}
$$

The solution of the above equation is:

$$
\begin{aligned}
e_{2}= & \alpha V^{2}+m g\left(\sin \vartheta+V \omega_{2}\right)+m(\cos \vartheta \\
& \left.+V \omega_{2}\right)+c_{1} e_{2}+\lambda_{1} e_{1}-c^{2}{ }_{1} e_{2}-c_{1} \lambda_{1} \zeta_{1}-\ddot{\vartheta}_{r}
\end{aligned}
$$

Using gyroscopes and accelerometers for attitude information fusion, the second Lyapunov function is selected as:

$$
V_{2}=V_{1}+\frac{1}{2} e_{2}^{2}
$$

Differentiate as:

$$
\dot{V}_{2}=\dot{V}_{1}+e_{1} \dot{e}_{2}
$$

Carry out nonlinear integral and replace it with:

$$
\begin{aligned}
\dot{V}_{2}= & e_{1} \dot{e}_{2}-c_{1} e_{2}^{2}-\lambda_{1} \zeta_{1} e_{1}+e_{2}\left[\alpha V^{2}\right. \\
& +m g\left(\sin \vartheta+V \omega_{2}\right)+m\left(\cos \vartheta+V \omega_{2}\right) \\
& \left.+c_{1} e_{2}+\lambda_{1} e_{1}-c^{2}{ }_{1} e_{2}-c_{1} \lambda_{1} \zeta_{1}-\ddot{\vartheta}_{r}\right]
\end{aligned}
$$

The equivalent control law of the sliding surface is constructed by linearizing the unmanned aerial vehicle's flight balance point, and the reference input parameters of the rudder angle are selected as:

$$
\begin{aligned}
\vartheta_{r}= & -\hat{\delta}\left(\alpha V^{2}+m g\left(\sin \vartheta+V \omega_{2}\right)+m(\cos \vartheta\right. \\
& \left.\left.+V \omega_{2}\right)+c_{1} e_{2}+\lambda_{1} e_{1}-c^{2}{ }_{1} e_{2}-c_{1} \lambda_{1} \zeta_{1}-\ddot{\vartheta}_{r}\right)
\end{aligned}
$$$$
\text { While } \vartheta= \pm 90^{\circ}, m\left(\cos \vartheta+V \omega_{2}\right)=0 \text {. }
$$

\subsection{Adaptive correction of flight parameters}

Based on the conventional synovial surface, an integral term of tracking error is added, and the adaptive law is designed as follows:

$$
V_{3}=V_{2}+\frac{\lambda_{1} \zeta_{1}^{2}}{2}+\frac{\lambda_{2} \zeta_{2}^{2}}{2}+\frac{\tilde{\delta}^{2}}{2 \varepsilon_{1} \delta}
$$

Then: 


$$
\dot{V}_{3}=\dot{V}_{2}+\lambda_{1} \zeta_{1} e_{1}+\lambda_{2} \zeta_{2} e_{2}-\frac{\tilde{\delta} \hat{\dot{\delta}}}{\varepsilon_{1} \delta}
$$

Through the fusion filtering of attitude information, the optimized flight parameter correction adaptive law of the unmanned aerial vehicle is obtained:

$$
\hat{\dot{\delta}}=\varepsilon_{1} \alpha V^{2} e_{2}
$$

An amendment is taken into the steady state error:

$$
\dot{V}_{3}=-c_{1} e_{1}^{2}-c_{2} e_{2}^{2} \leq 0
$$

Under the condition of constant dynamic acceleration, the fusion error of UAV flight at two times is obtained. According to Barbalat's theorem, it is known that:

$$
\lim _{t \rightarrow \infty} e_{1}=\lim _{t \rightarrow \infty} e_{2}=0
$$

It can be proved that the UAV can track the reference signal adaptively under the steady state error correction control and the adaptive law, and the tracking error converges globally to zero, thus improving the flight stability.

\section{ANALYSIS OF SIMULATION EXPERIMENT}

In order to test the application performance of this method in the flight stability control of UAV, the simulation experiment is carried out, and the control algorithm is programmed by Matlab. UAV quality set as $150 \mathrm{Kg}$, moment of inertia is $2.4 \mathrm{rad} / \mathrm{s}$, the signal - to noise ratio of the disturbance of airflow disturbance is $-10 \mathrm{~dB}$, the number of shots is 1000 , adaptive parameters for control instruction transmission $\varepsilon_{1}=0.1$, select Sliding Mode Control Parameters $\lambda_{1}=1, \lambda_{2}=1, c_{1}=2, c_{2}=2$, initial value of steady state error $\hat{\delta}_{0}=-15$, Inertial integral coefficient . Based on the above simulation environment and parameter design, we carry out simulation analysis of UAV flight stability control, and get the error convergence curve of UAV attitude parameter which is controlled by this method and traditional method, as shown in Figure 1.

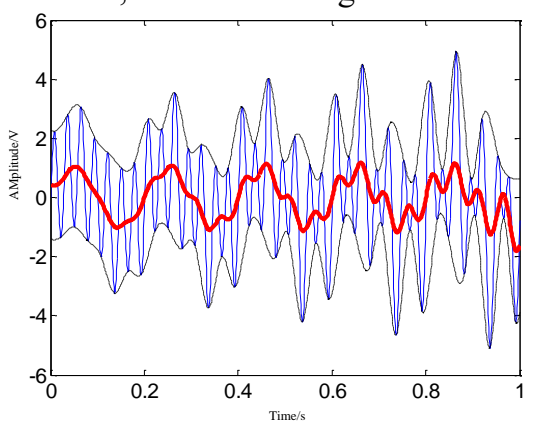

(a) Conventional method

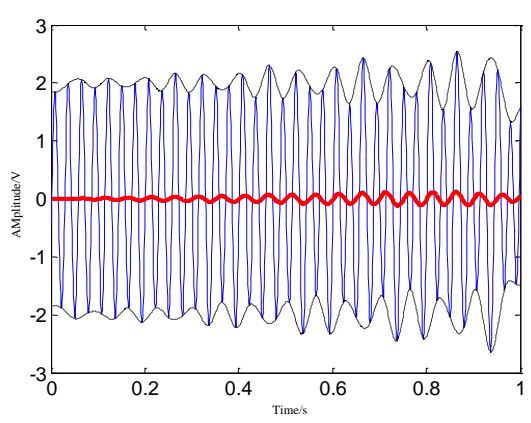

(b)This method

Fig. 1 Attitude parameter convergence curve of unmanned aerial vehicle flight stability control

Figure 1 shows that the proposed method is used to control the flight stability of UAV, and the oscillation amplitude of attitude parameter is small. Figure 2 describes the characteristic distribution of UAV flight stability control using this method. The analysis shows that the characteristic distribution of UAV flight control using this method is stable. It shows that the flight stability control ability is strong and the steady-state error is effectively suppressed.



Fig. 2 Characteristic distribution state of unmanned aerial vehicle flight stability control

\section{CONCLUSION}

In the course of flight, the UAV is affected by the disturbed airflow and the aircraft itself, and under the disturbance of uncertain climatic conditions, which results in the instability of the flight attitude and the steady state error. In order to reduce the steady - state error of UAV flight, a flight stability control method based on steady - state error correction is proposed in this paper. The simulation results show that the stability of the flight control of the UAV is better by the method, and the self - adaptive correction capability of the flight state parameters is strong. The flight stability of UAV is guaranteed.

\section{ACKNOWLEDGMENTS}

This project is supported by Foundation for Distinguished Young Talents (Natural Science, 2015KQNCX218) 


\section{REFERENCES}

1. DI Bin ZHOU Rui DONG Zhuo-ning. Cooperative localization and tracking of multiple targets with the communication-aware unmanned aerial vehicle system. Control and Decision, 2016, 31(04): 616-622.

2. Aguila-Camacho $\mathrm{N}$, Duarte-Mermoud $\mathrm{M}$ A, Gallegos J A. Lyapunov functions for fractional order systems $[\mathrm{J}]$. Communications in Nonlinear Science and numerical Simulation, 2014, 19(9): 2951-2957.

3. LU Xing hua .Anti Interference Control Algorithm for UAV Based on Attitude Fusion Filter [J]. Transducer and Microsystem Technologies, 2016,35(7):116-119.

4. Lu Xinghua, Zhen Hanjian, Duan Wuxing.Fault Tolerant Controller Design for Embedded Multi Mode Control System [J]. Machinery \& Electronics, 2016,34(4): 62-65.

5. WANG Xun, ZHANG Daibing, SHEN Lincheng. A Virtual Force Based Path Following Approach for Unmanned Aerial Vehicles. ROBOT, 2016, 38(3): 329-336.
6. BROX T and MALIK J. Large displacement optical flow: descriptor matching in variational motion estimation[J]. IEEE Transactions on Pattern Analysis and Machine Intelligence, 2010, 33(3): 500-513.

7. EVANGELIO R H, PATZOLD M, and KELLER I. Adaptively splitted GMM with feedback improvement for the task of background subtraction[J]. IEEE Transactions on Information Forensics and Security, 2014, 9(5): 863-874.

8. LIU Kai, ZHU Ji-hong, YU Bo. Longitudinal control of aircraft with thrust vectoring using robust dynamic inversion [J]. Control and Decision, 2013, 28(7): 1113-1116.

9. DOU Huijing, WANG Qianlong, ZHANG Xue. A Joint Estimation Algorithm of TDOA and FDOA Based on Wavelet Threshold De-noising and Conjugate Fuzzy Function. JEIT, 2016, 38(5): 1123-1128.

10. Lu Xinghua, Zhan Shiwei, Yu Wenquan.Heading Correction Control Algorithm for UAV in Steepest Glide Trajectory [J].Computer \& Digital Engineering, 2016,44(12): 2370-2374,2425. 